\title{
Research on the Influence of Exhaust Temperatures on the Emission Performance of Urea-SCR After-treatment System
}

\author{
WANG Jing ${ }^{1,2, a}$, WANG Qian ${ }^{1, \text { b }}$, WU Li-cheng ${ }^{1}$, ZHAO Wei $^{1}$, LI Shuo $^{1}$ \\ ${ }^{1}$ School of Energy and Power Engineering, Jiangsu University, Jiangsu, 212013, China \\ ${ }^{2}$ Zhenjiang Watercraft College, Jiangsu, 212003, China \\ akingarrow10@163.com, bqwang@ujs.edu.cn
}

Keywords: Diesel, NOx Emission, SCR, Numerical Simulation

\begin{abstract}
SCR (Selective Catalytic Reduction) technology is the most effective after-treatment technology of DeNOx on vehicle diesel engines. In this paper, a modeling of vehicle diesel Urea-SCR after-treatment system was set up with the method of CFD coupled with chemical reaction dynamic, to make a full-scale simulation and comparison of two Urea-SCR catalyst systems, analyzing the influences of different exhaust temperatures on the emission performance of SCR system, so as to better improve the design of SCR system.
\end{abstract}

\section{Introduction}

Due to the changeable operating conditions and exhaust parameters of the vehicle diesel, its NOx emission has become one of the main atmospheric pollutants in recent years. The whole world has been looking for solutions of the problem, and the Urea-SCR (Urea Selective Catalytic Reduction) technology is the relatively mature and widely-used one[1]. As the structure of the SCR system has great influence on the reduction of NOx emission, this paper made a detailed full-scale 3D numerical simulation of two Urea-SCR modeling structures with AVL FIRE software, to analyze the characters of the inner flow field distribution and the influence of exhaust temperature on SCR system, so as to provide a theoretical background for the structure design and optimization of the SCR system.

\section{Numerical Simulation Model}

It is a Urea-SCR system simulation of a home-made six-cylinder vehicle engine on AVL FIRE platform. The main tasks include urea solution spray, flow in porous medium and catalytic reaction.

Urea Solution Spray Model. The Discrete Droplet Model (DDM) is used in the urea solution spry simulation in this paper. It regards the urea droplet as discrete ones once they leave the nozzle, and makes use of the Lagrangian method and Euler's method to figure out the motion track of the droplets. The secondary breaking process of the droplets is simulated by HuhGosman break-up model[2].

Model of the Chemical Reaction between Carrier and Catalyst Surface. As the ceramic honeycomb carrier is too small in size and too large in number, it is very difficult to directly simulate the three-dimensional flow in the channel, and the inner flow in the carrier has to be simplified. In this paper, the integral ceramic honeycomb catalyst carrier is simplified to porous medium in single direction. The pressure loss is calculated by Forchheimer model[3].

$$
\frac{\mathrm{d} p_{g}}{\mathrm{dx}_{\mathrm{i}}}=-\alpha_{i} \cdot \mu \cdot w_{i}-\zeta \cdot \frac{\rho}{2} \cdot|w| w_{i}
$$

In this paper, the chemical reaction occurred on the catalyst surface mainly involves the following five equations:

$$
\begin{aligned}
& \mathrm{HCNO}+\mathrm{H}_{2} \mathrm{O} \rightarrow \mathrm{NH}_{3}+\mathrm{CO}_{2} \\
& 4 \mathrm{NH}_{3}+4 \mathrm{NO}+\mathrm{O}_{2} \rightarrow 4 \mathrm{~N}_{2}+6 \mathrm{H}_{2} \mathrm{O}
\end{aligned}
$$




$$
\begin{aligned}
& 2 \mathrm{NH}_{3}+\mathrm{NO}+\mathrm{NO}_{2} \rightarrow 2 \mathrm{~N}_{2}+3 \mathrm{H}_{2} \mathrm{O} \\
& 8 \mathrm{NH}_{3}+6 \mathrm{NO}_{2} \rightarrow 7 \mathrm{~N}_{2}+12 \mathrm{H}_{2} \mathrm{O} \\
& 4 \mathrm{NH}_{3}+3 \mathrm{O}_{2} \rightarrow 2 \mathrm{~N}_{2}+6 \mathrm{H}_{2} \mathrm{O}
\end{aligned}
$$

Equation 2 means the isocyanic acid, the thermal decomposition product of urea, has a hydrolysis reaction with vapor on the surface of the catalyst, and generates the ammonia gas and carbon dioxide. Equations 3 to 5 involve the $\mathrm{SCR}$ of $\mathrm{NH}_{3}$ to $\mathrm{NO}_{\mathrm{X}}$. As $\mathrm{NO}$ is the main nitrogen oxide in the exhaust of engine (about 90\%), the reaction 3 is called the standard SCR. Studies show that the existence of $\mathrm{NO}_{2}$ can increase the reaction speed. When $\mathrm{V}\left(\mathrm{NO}_{2}\right) / \mathrm{V}\left(\mathrm{NO}_{\mathrm{X}}\right) \approx 50 \%$, the reaction speed is the fastest. Therefore, equation 4 is called the fast SCR. When the ratio of $\mathrm{V}\left(\mathrm{NO}_{2}\right) / \mathrm{V}\left(\mathrm{NO}_{\mathrm{X}}\right)$ continues to increase, the slow SCR shown in equation 5 is in dominance, and the conversion efficiency of $\mathrm{NO}_{\mathrm{X}}$ is reduced[4]. Equation 6 is the oxidation reaction of ammonia gas, in order to prevent the ammonia leakage.

\section{Evaluating Indicators of SCR System}

SCR Conversion Efficiency. The conversion efficiency of SCR system is a direct reflection of its performance. The definition of SCR conversion efficiency is as follows:

$$
\eta_{i}=\frac{C_{i, \text { in }}-C_{i, \text { out }}}{C_{i, \text { in }}} \times 100 \%
$$

Uniformity Coefficient. The speed at the catalyst inlet and the distribution of reductants may directly affect the conversion efficiency of the catalyst. The nonuniformity of flow speed may not only increase the flow resistance, but also reduce the $\mathrm{NO}_{\mathrm{x}}$ conversion efficiency and accelerate the deterioration of catalyst, shortening the lifespan of catalyst. What's more, the nonuniformity of reductants may cause an excess or lack of reductants in local areas, resulting in a decrease of SCR conversion efficiency and ammonia leakage[5].

The uniformity coefficient $\gamma$ is defined as[6]:

$$
\gamma=1-\frac{1}{2 n} \sum_{i=1}^{n} \frac{\sqrt{\left(c_{i}-\bar{c}\right)^{2}}}{\bar{c}}
$$

\section{CFD Modeling of Urea-SCR Catalyst}

Object of Research. The object of this research is the SCR system in a six-cylinder diesel engine. The main performance index of the diesel engine is shown in Tab 1. The reductant used in the SCR system is urea solution with the mass fraction of $32.5 \%$. The catalyst is in the form of a barrel, $350 \mathrm{~mm}$ in length and $250 \mathrm{~mm}$ in diameter, with the inlet and outlet of $100 \mathrm{~mm}$ in diameter. Monolithic catalysts $\mathrm{V}_{2} \mathrm{O}_{5} / \mathrm{WO}_{3} / \mathrm{TiO}_{2}$ are used. The inner part is in honeycomb structure with square holes. The volume is $14 \mathrm{~L}$, the hole density is 62 holes $/ \mathrm{cm}^{2}$, the wall thickness is $0.2 \mathrm{~mm}$, and the thickness of coating layer of the carrier is $0.127 \mathrm{~mm}$.

Table 1 Performance Parameter of the Diesel Engine

\begin{tabular}{cc}
\hline Item & Parameters \\
\hline Type & six-cylinder, inline, water-cooled, four-stroke \\
Intake System & turbocharged, intercooled \\
Fuel System & high pressure common rail electronic control \\
Displacement $[\mathrm{L}]$ & system \\
Rated Power[kW]/ (Rotational Speed $[\mathrm{r} / \mathrm{min}])$ & 6.5 \\
Maximum Torque/(Rotational Speed[r/min]) & $177(2300)$ \\
\end{tabular}


Modeling Plan. Two plans were applied in this article. In the first plan, a three-hole nozzle was used, with the spray angle being $10^{\circ}$ and the horizontal angle being $120^{\circ}$. The distance between the injection point of urea and the end surface of catalyst is $630 \mathrm{~mm}$. Restrained by the mixture condition of urea and the exhaust as well as the workmanship standard, the urea spray angle was set as $30^{\circ}$. The geometric model is shown in Fig 1 . Since its structure is not very complicated, the grid model is also generated by ICEMCFD as is shown in Fig 2.

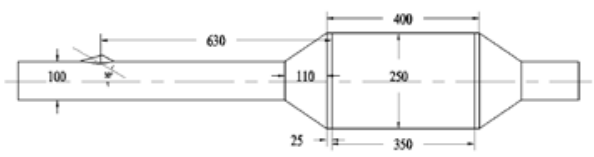

Fig. 1 Geometric Model of Catalyst

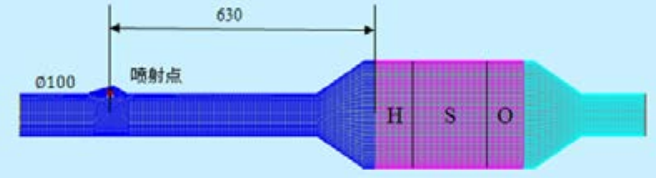

Fig. 2 Grid Model

In the second plan, a four-hole nozzle was used to spray radially in one plane, with the included angle of adjacent holes being $90^{\circ}$. The distance between the nozzle and the center of the catalyst inlet is described as $7 \mathrm{~d}$. Plenty of tiny holes with the diameter of $8 \mathrm{~mm}$ are uniformly distributed around the diffuser in the catalyst. The geometric model is shown in Fig 3. Due to its complicated structure, FAME in FIRE is used to make automatic meshing for it and encrypt the holes on the diffuser at the same time, so as to ensure the accuracy of calculation. The grid generated is shown in Fig 4.

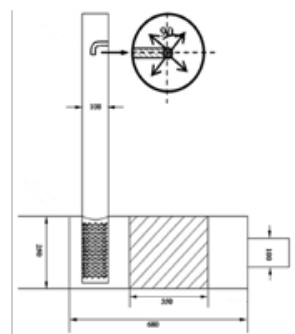

Fig. 3 Catalyst Structure of SCR System

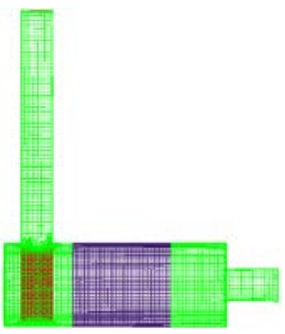

Fig. 4 Computational Grid of SCR Catalyst

Boundary Condition. Three typical operating conditions A25, B50, C100 are chosen from the 13 operating points defined by GB17691-2005 automobile emission limits and methods of measurement (Phase III, IV and V of National Standard) as the input value of boundary conditions, shown in Tab 2.

Table 2 Boundary Conditions of Typical Operating Conditions

\begin{tabular}{ccccc}
\hline $\begin{array}{c}\text { Operating } \\
\text { Conditions }\end{array}$ & $\begin{array}{c}\text { Exhaust Flow } \\
\text { Discharge[kg/h] }\end{array}$ & $\begin{array}{c}\text { Speed } \\
{[\mathrm{m} / \mathrm{s}]}\end{array}$ & $\begin{array}{c}\text { Exhaust } \\
\left.\text { Temperature[ }{ }^{\circ} \mathrm{C}\right]\end{array}$ & $\begin{array}{c}\varphi\left(\mathrm{NO}_{\mathrm{X}}\right) \\
{[\mathrm{ppm}]}\end{array}$ \\
\hline A25 & 400 & 25 & 281 & 504 \\
B50 & 694 & 41 & 330 & 721 \\
C100 & 971 & 69 & 428 & 1109 \\
\hline
\end{tabular}

\section{Influence of the Exhaust Temperature}

The exhaust temperature may not only affect the reaction rate of SCR, but also affect the evaporation and thermolysis of urea solution and the generation of reductants. Generally speaking, the higher the exhaust temperature is, the stronger the catalytic activity will be, and the faster the reaction rate will be. However, if the exhaust temperature is too high, the catalytic activity may be reduced, and phenomena like catalyst sintering and high temperature ageing may appear, which may in turn reduce the conversion efficiency of $\mathrm{NO}_{\mathrm{X}}$. The range of the exhaust temperature of diesel at the actual load is between 150 and $700^{\circ} \mathrm{C}$ [7]. But for the vanadium-base SCR catalyst, the activation temperature range is between 300 and $450^{\circ} \mathrm{C}$. Therefore, this essay only analyzes the influences of different exhaust temperatures $\left(250^{\circ} \mathrm{C}, 300^{\circ} \mathrm{C}, 350^{\circ} \mathrm{C}, 400^{\circ} \mathrm{C}, 450^{\circ} \mathrm{C}\right.$ and $500^{\circ} \mathrm{C}$ ) on SCR system in two plans under B50 operating condition. 


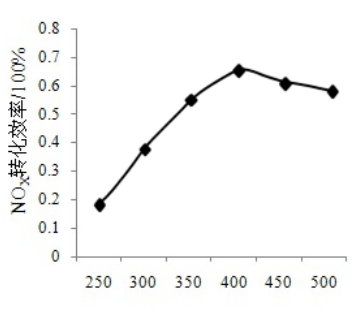

(a)

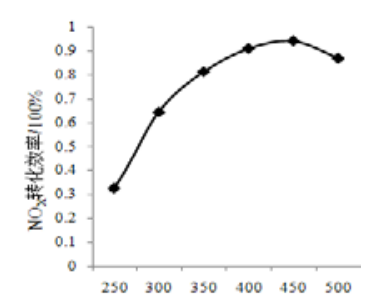

(b)

Fig. 5 Change of $\mathrm{NO}_{\mathrm{X}}$ Conversion Efficiency under Different Exhaust Temperatures

Fig. 5(a) is the curve that shows the change of $\mathrm{NO}_{\mathrm{X}}$ conversion efficiency under different exhaust temperatures in Plan One. As is shown in the figure, the exhaust temperatures have great influence on the conversion efficiency of $\mathrm{NO}_{\mathrm{X}}$. In the range of $250-400^{\circ} \mathrm{C}$, the $\mathrm{NO}_{\mathrm{X}}$ conversion efficiency is proportional to the temperature, and it will reach the maximal value of $66 \%$ at $400^{\circ} \mathrm{C}$. However, when the temperature is higher than $400^{\circ} \mathrm{C}$, the $\mathrm{NO}_{\mathrm{X}}$ conversion efficiency will begin to drop with the rising of temperature after it reaches the maximal value.

Fig. 5(b) is the curve that shows the change of $\mathrm{NO}_{\mathrm{X}}$ conversion efficiency under different exhaust temperatures in Plan Two. As is shown in the figure, the exhaust temperatures have great influence on the conversion efficiency of $\mathrm{NO}_{\mathrm{X}}$. In the range of $250-450^{\circ} \mathrm{C}$, the $\mathrm{NO}_{\mathrm{X}}$ conversion efficiency is proportional to the temperature, and it will reach the maximal value of $94 \%$ at $4500^{\circ} \mathrm{C}$. However, when the temperature is higher than $450^{\circ} \mathrm{C}$, the $\mathrm{NO}_{\mathrm{X}}$ conversion efficiency will begin to drop with the rising of temperature after it reaches the maximal value.

\section{Conclusions}

Due to the large amount of exhaust flow of diesel engines, most exhaust gas is in turbulent motion in SCR system, which determines the spatial distribution of most parameters in the exhaust pipe. This article analyzes the influence of different exhaust temperatures on the emission performance of two SCR systems under B50 operating condition. The result shows that, the variation trends of the $\mathrm{NO}_{\mathrm{X}}$ conversion efficiency in two plans are similar in that both rise with the rising of temperature to peak values, and then drop afterwards. The reasons are as follows. When the temperature is very low, the evaporation and thermolysis of urea as well as the $\mathrm{NH}_{3}$ generated in the hydrolysis reaction of isocyanic acid in the catalyst are very low. As a result, the $\mathrm{NO}_{\mathrm{X}}$ conversion efficiency is very low. Then, with the rising of the temperature, the speed of the evaporation and thermolysis of urea droplets are increased and more reductants are generated. At the same time, the activity of catalyst also increased with the rising of temperature, which is suitable for the full reaction of SCR and lead to the rise of $\mathrm{NO}_{\mathrm{X}}$ conversion efficiency. However, with the further rising of the temperature, the activity of catalyst begins to decline. The chances of oxidizing reaction between $\mathrm{NH}_{3}$ and $\mathrm{O}_{2}$ increase, while the amount of $\mathrm{NH}_{3}$ reacted with $\mathrm{NO}_{\mathrm{X}}$ begins to drop. As a result, the $\mathrm{NO}_{\mathrm{X}}$ conversion efficiency begins to decline. In Plan One, the maximal value of $\mathrm{NO}_{\mathrm{X}}$ conversion efficiency reaches $66 \%$ at the temperature of $400^{\circ} \mathrm{C}$, while in Plan Two, the maximal value reaches $94 \%$ at the temperature of $450{ }^{\circ} \mathrm{C}$, which means the $\mathrm{NO}_{\mathrm{X}}$ conversion efficiency in Plan Two is higher than that in Plan One. The reason lies in that a diffuser with plenty of tiny holes distributed around is adopted in Plan Two. When the urea droplets move with the flow of exhaust gases, they will evaporate and break up, and both the diameters and quantities of droplets will reduce in this way. When they reach the enclosed bottom of the diffuser, they will be rebounded due to the inertia caused by the movement of exhaust gases when touching the bottom, which may accelerate the breaking up of droplets. When the gases flow out of the diffuser, the speed will increase evidently, and this may further accelerate the evaporation and breaking up of droplets. Although some small whirlpools may be generated when the flow enters the front part of the catalyst, the general distribution of the flow is relatively uniform and the concentration distribution of reductants in the catalyst is relatively uniform. All these are helpful to improve the conversion efficiency of the SCR system. 


\section{Acknowledgement}

In this paper, the research was supported by the Key Project of National Natural Science Foundation of China (Project No. 51276084), the Industrial Support Project of Jiangsu province(Project No. BE2010198), the Cooperative Innovation fund of Jiangsu Province (Project No. BY2013065-02), the Priority Academic Program Development of Jiangsu Higher Education Institutions and the Innovative Research Program of Graduate Students in Jiangsu Higher Education Institutions (Project No. CXZZ11_0560).

\section{References}

[1] M. Koebel, M. Elsener, M. Kleemann. Urea-SCR: a promising technique to reduce $\mathrm{NO}_{\mathrm{X}}$ emissions from automotive diesel engines[J]. Catalysis Today, 2000, 59 (3-4):335-345.

[2] AVL List GmbH. FIRE v2011 - Lagrangian Multiphase Module [Z]. Graz: 2011.

[3] Vishal A.Jambhekar. Forchheimer Porous-media Flow Models - Numerical Investigation and Comparison with Experimental Data[D]. Stuttgart: University of Stuttgart , 2011.

[4] Cristian Ciardelli, Isabella Nova, Enrico Tronconi, et al. Reactivity of $\mathrm{NO} / \mathrm{NO}_{2}-\mathrm{NH}_{3}$ SCR system for diesel exhaust aftertreatment : Identification of the reaction network as a function of temperature and $\mathrm{NO}_{2}$ feed content[J]. Applied Catalysis B: Environmental, 2007, 70 (1-4):80-90.

[5] Howitt J S, Sekella T C. Flow Effects in Monolithic Honeycomb Automotive Catalytic Converters. SAE Paper 740244.

[6] Weltens H, Bressler $\mathrm{H}$, Terres $\mathrm{F}$, et al. Optimization of Catalytic Converter Gas Flow Distribution by CFD Prediction[C]. SAE Paper, 930780.

[7] Chang Shiying, Yang Dongxia, Duan Chunkun, etc. The Development of the Technology of $\mathrm{NH}_{3}$ Selective Catalytic Reduction of $\mathrm{NO}_{\mathrm{X}}$ in Diesel Vehicles [J]. Environmental Science \& Technology, 2009(10): 97-102. 